\title{
A semiclassical generalized quantum master equation for an arbitrary system-bath coupling
}

\author{
Qiang Shi and Eitan Geva ${ }^{\text {a) }}$ \\ Department of Chemistry and the FOCUS Center, University of Michigan, Ann Arbor, Michigan 48109-1055
}

(Received 23 December 2003; accepted 15 March 2004)

\begin{abstract}
The Nakajima-Zwanzig generalized quantum master equation (GQME) provides a general, and formally exact, prescription for simulating the reduced dynamics of a quantum system coupled to a, possibly anharmonic, quantum bath. In this equation, a memory kernel superoperator accounts for the influence of the bath on the dynamics of the system. In a previous paper [Q. Shi and E. Geva, J. Chem. Phys. 119, 12045 (2003)] we proposed a new approach to calculating the memory kernel, in the case of arbitrary system-bath coupling. Within this approach, the memory kernel is obtained by solving a set of two integral equations, which requires a new type of two-time system-dependent bath correlation functions as input. In the present paper, we consider the application of the linearized semiclassical (LSC) approximation for calculating those correlation functions, and subsequently the memory kernel. The new approach is tested on a benchmark spin-boson model. Application of the LSC approximation for calculating the relatively short-lived memory kernel, followed by a numerically exact solution of the GQME, is found to provide an accurate description of the relaxation dynamics. The success of the proposed LSC-GQME methodology is contrasted with the failure of both the direct application of the LSC approximation and the weak coupling treatment to provide an accurate description of the dynamics, for the same model, except at very short times. The feasibility of the new methodology to anharmonic systems is also demonstrated in the case of a two level system coupled to a chain of Lennard-Jones atoms. (C) 2004 American Institute of Physics.
\end{abstract} [DOI: 10.1063/1.1738109]

\section{INTRODUCTION}

Quantum effects play a central role in a variety of important processes that take place in condensed phase environments. ${ }^{1-3}$ Hence, the simulation of quantum dynamics in condensed phase hosts is one of the most important challenges facing theoretical chemistry. Whereas numerically exact classical molecular dynamics simulations are feasible for relatively complex many-body systems, the analogous numerically exact solution of the Schrödinger equation ${ }^{4-8}$ for such systems remains far beyond the reach of currently available computer resources, due to the exponential scaling of the computational effort with the number of degrees of freedom (DOF).

A common approach for dealing with this difficulty is based on the observation that, in practice, one can often directly probe and/or manipulate only a small number of the DOF. The subsystem subject to direct observation and/or manipulation may correspond to the reaction coordinate, a relaxing vibrational mode of a solute molecule, or an optically active transition in a solvated chromophore molecule. Thus, it is worthwhile to consider a strategy that combines an accurate description of the subsystem, which will be referred to as the system from now on, with a minimal, yet accurate, treatment of the rest of the DOF, which will be referred to as the bath. The key to the success of such an approach relies on one's ability to accurately filter out those aspects of the many-body bath dynamics which affect the system. It should

${ }^{a)}$ Electronic mail: eitan@umich.edu be noted that this point of view is analogous to that taken in many experiments, where the environment DOF are probed via their impact on the observed system. Thus, this approach has the additional advantage of isolating those aspects of the bath dynamics which are probed by performing measurements on the system.

Let us consider a general system, where the overall quantum-mechanical Hamiltonian has been separated into the following four generic terms: (1) The system Hamiltonian, $\hat{H}_{s}$, which only depends on the system DOF; (2) The bath Hamiltonian, $\hat{H}_{b}$, which only depends on the bath DOF; (3) The system-bath coupling, $\hat{H}_{\mathrm{bs}}$; and (4) $\hat{W}(t)$, which stands for an external perturbation that the system is subject to, and which can be described in terms of system operators and explicitly time-dependent classical fields

$$
\hat{H}=\hat{H}_{s}+\hat{H}_{b}+\hat{H}_{\mathrm{bs}}+\hat{W}(t) .
$$

The available theoretical approaches for dealing with the system dynamics can then be classified based on the assumptions they make with respect to $\hat{H}_{\mathrm{bs}}$ and $\hat{W}(t)$ (cf. Fig. 1). In the absence of an external perturbation, i.e., $\hat{W}(t)=0$, the overall system (the system plus the bath) will be at a state of equilibrium, which is described by a density operator of the form $\hat{\rho}=e^{-\beta \hat{H}} / \operatorname{Tr}\left[e^{-\beta \hat{H}}\right] \quad\left(\beta=1 / k_{B} T\right.$, where $k_{B}$ is Boltzmann's constant and $T$ is the absolute temperature). Most experiments start with the overall system in this state. The scaling of the computational effort involved in computing quantum mechanical expectation values at such an equilib- 


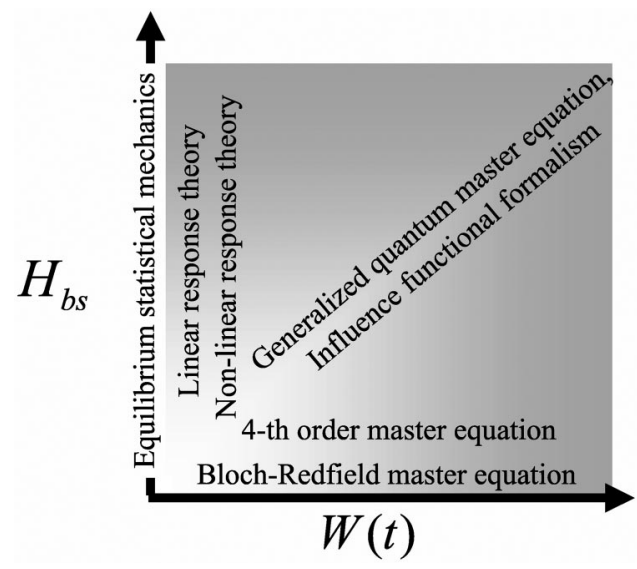

FIG. 1. A classification of theoretical approaches based on the assumptions they make with respect to the strength of the system-bath coupling, $\hat{H}_{\mathrm{bs}}$, and external perturbation, $\hat{W}(t)$.

rium state is favorable, and they can be calculated via powerful imaginary-time path integral techniques, for relatively complex many-body systems. ${ }^{9,10}$

Linear response theory (LRT) is applicable in the case where $\hat{W}(t)$ is finite, but can be treated as a small perturbation. It corresponds to the region that lie close to the origin and along the $\hat{H}_{\text {bs }}$ axis in Fig. 1. In this case, the nonequilibrium relaxation dynamics of the overall system is the same as that of its spontaneous fluctuations around equilibrium, and can be described in terms of two-time correlation functions $(\mathrm{CFs}) .{ }^{11}$ It should be noted that LRT does not require that we explicitly divide the overall system into a system and a bath, and is in fact valid for an arbitrary system-bath coupling. At the same time, the earlier mentioned CFs represent much more reduced quantities in comparison to the full wave function or density matrix of the overall system. As such, they may be thought of as filtering out those aspects of the overall system dynamics which are relevant for describing the relaxation process. LRT is particularly useful in two situations: (1) When the system is subject to a relatively weak external perturbation, which shifts the overall system only slightly relative to its equilibrium state; (2) When the system follows rate kinetics, such that the rate constant does not depend on the initial state, and can therefore be conveniently calculated with an initial state which is in the close vicinity of equilibrium. An important example for the first scenario is provided by linear spectroscopy, where the laser field is often treated as a small perturbation. For example, the absorption spectrum of a chromophore in solution can be expressed in terms of a two-time dipole CF. In fact, this approach can be extended so as to account for higher order nonlinear response to the laser field, and put it in terms of multitime dipole CFs. ${ }^{12}$ However, it should be noted that such multitime CFs become increasingly more difficult to compute as one moves further and further away from equilibrium. An important example of the second scenario is given by chemical reactions, where rare event statistics associated with barrier crossing leads to rate kinetics, and where the rate constant can be expressed in terms of a two-time $\mathrm{CF}$ which involves the reactive flux operator. ${ }^{13,14}$
Another important class of methods is based on the explicit division of the overall system into a system and a bath, and corresponds to the case where $\hat{H}_{\text {bs }}$ can be treated as a small perturbation. This corresponds to the region that lies along, and in the close vicinity of, the $\hat{W}(t)$ axis in Fig. 1 . The assumption of weak coupling (WC) between the system and the bath, augmented with the complementary assumption that the system's relaxation occurs on a time scale which is much longer than that of the bath fluctuations, then leads to a description of the system dynamics by a Markovian quantum master equation (QME) of the Bloch-Redfield type. ${ }^{11,15-39}$ In this case, the influence of the bath shows up via population and phase relaxation rate constants, which can be expressed in terms of two-time free-bath CFs. In principle, the QME approach can be extended so as to take into account higher order terms in the perturbation expansion with respect to the system-bath coupling. ${ }^{26,40-43}$ However, the latter are given in terms of multitime $\mathrm{CFs}$, and are difficult to compute in practice.

The availability of feasible methods drops rapidly as one moves into the region where neither $\hat{W}(t)$ nor $\hat{H}_{\text {bs }}$ can be treated as small perturbations (cf. Fig. 1). One approach, which gained popularity over the last several decades, is based on the path integral formulation of quantum mechanics, ${ }^{44-46}$ and introduces the influence of the bath in terms of an influence functional (IF). ${ }^{47}$ One of the most important advantages of this approach has to do with the fact that the exact IF can be obtained in closed form, in the case of linear coupling to a harmonic bath. ${ }^{48-51}$ This fact, in conjunction with important algorithmic advances, such as the development of iterative tensor quasiadiabatic propagators by Makri and co-workers, have opened the door to numerically exact calculations of the reduced dynamics of this type of systems (as long as one can evaluate the remaining path integral over the system DOF). ${ }^{52-67}$ However, there are many important systems, e.g., liquid solutions, where it is difficult, and perhaps even impossible, to map the bath Hamiltonian onto a harmonic one. Recent attempts by Makri and coworkers to use semiclassical approximations in order to evaluate the IF in the case of anharmonic baths and nonlinear coupling ${ }^{68-70}$ appear promising, and their relationship to the present work will be discussed in Sec. V.

An alternative to the IF approach may be based on the equation of motion that governs the system dynamics, which is known as the generalized quantum master equation (GQME). ${ }^{16,17,71-77}$ The Nakajima-Zwanzig GQME $^{16,17,20-24,71-78}$ represents such an exact equation of motion. In this equation, the influence of the bath on the system is given in terms of a memory kernel superoperator. The latter is analogous to the IF in the sense that it contains all the information needed in order to account for the influence of the bath on the system dynamics. However, the use of this GQME, as such, has been rather limited due to difficulties in evaluating the memory kernel. As a result, the GQME has been mostly used as the starting point of more approximate treatments. The most popular approximation is based on the assumption that the system is weakly coupled to the bath, and leads to the earlier mentioned QME of the Bloch-Redfield type. Unfortunately, the very same WC as- 
sumption imposes serious restrictions on the range of phenomena that can be described by QMEs, and especially so in condensed phase systems. For example, QMEs are unable to describe such important phenomena as solvation dynamics and solvent memory effects, which are central to solution chemistry.

In a previous paper, ${ }^{78}$ we presented a new framework for calculating the Nakajima-Zwanzig memory kernel without resorting to the assumption of weak system-bath coupling. The strategy we proposed is based on expressing this kernel in terms of two-time system-dependent bath correlation functions (SDBCFs), which should be contrasted with the freebath CFs that the WC approach gives rise to. The transition from bath-free to system-dependent CFs reflects the fact that one has to account for the reverse action of the system on the bath, which remains unaccounted for within the framework of the WC approach. It should be noted that properly accounting for this feedback reaction is crucial for describing such important phenomena as solvation dynamics.

A demonstration of the new approach in the case of a two-level system linearly coupled to a harmonic bath has been provided in Ref. 78. In this case, it was possible to compute the exact SDBCFs, and hence, the exact memory kernel. However, in the more general case of nonlinear coupling to an anharmonic bath, one would have to develop feasible and reliable approximate schemes for calculating the sought after SDBCFs. In the present paper, we consider a methodology which will be applicable within this more general scenario.

Several strategies have been proposed over the last two decades, that attempt to address the challenge of providing an effective, computationally feasible, and versatile approximate method for calculating quantum-mechanical CFs. Those methods are based on various approaches, including a mixed quantum-classical treatment, ${ }^{79-84}$ analytical continuation, ${ }^{85-94}$ centroid molecular dynamics, ${ }^{14,95-111}$ quantum mode coupling theory, ${ }^{93,112-115}$ and the semiclassical approximation. ${ }^{2,68,116-133}$ From those, semiclassical and analytical continuation methods appear to be the most suitable for calculating the SDBCFs. Since $\sim N^{4}$ SDBCFs are required for calculating the memory kernel, it is important to choose the most cost-effective method (as long as it is reliable!). In this paper we consider the application of the linearized semiclassical (LSC) method, which is very flexible and relatively inexpensive, to the calculation of the Nakajima-Zwanzig memory kernel. It is important to note that the LSC approximation, like many of the other approximate methods mentioned earlier, works better at short times. Thus, while LSC will probably fail when applied directly to simulate the system dynamics, one expects it to do much better if it is only used for calculating the memory kernel, which is often relatively short lived, followed by solving the Nakajima-Zwanzig GQME in a numerically exact manner.

The structure of the remainder of this paper is as follows: The theoretical framework for the Nakajima-Zwanzig memory kernel is outlined in Sec. II. The application of the LSC approximation to the calculation of the this memory kernel is considered in Sec. III. The methodology is tested on a benchmark spin-boson problem and its feasibility is dem- onstrated in the case of an anharmonic bath model in Sec. IV. The main conclusions are summarized and discussed in Sec. V.

\section{THE MEMORY KERNEL}

In this section we provide an overview of our recently proposed approach to calculating the memory kernel of the Nakajima-Zwanzig GQME, for an arbitrary system-bath coupling. The formalism presented herein is based on a somewhat more general treatment of the system-bath coupling term, but is otherwise similar to that in Ref. 78 (where a more detailed discussion of other aspects of the theory has been provided).

We consider an overall system with the following general quantum-mechanical Hamiltonian

$$
\hat{H}=\hat{H}_{s}+\hat{H}_{b}+\hat{H}_{\mathrm{bs}},
$$

where $\hat{H}_{s}, \hat{H}_{b}$, and $\hat{H}_{\mathrm{bs}}$ are as in Eq. (1) $[\hat{W}(t)$ may be added at a later stage via the Hamiltonian term in the GQME]. The initial state of the overall system is assumed to have the following factorized form:

$$
\hat{\rho}(0)=\hat{\rho}_{s}(0) \otimes \hat{\rho}_{b}^{\mathrm{eq}},
$$

where $\hat{\rho}_{s}(0)$ is the initial density operator of the system, and

$$
\hat{\rho}_{b}^{\mathrm{eq}}=e^{-\beta \hat{H}_{b}} / \operatorname{Tr}_{b}\left[e^{-\beta \hat{H}_{b}}\right]
$$

is the density operator of the free bath at thermal equilibrium ( $\operatorname{Tr}_{b}$ stands for partial trace with respect to the Hilbert space of the bath). It is also assumed, without loss of generality, that

$$
\left\langle\hat{H}_{\mathrm{bs}}\right\rangle_{\mathrm{eq}}^{0} \equiv \operatorname{Tr}_{b}\left[\hat{\rho}_{b}^{\mathrm{eq}} \hat{H}_{\mathrm{bs}}\right]=0 .
$$

As is well known, the reduced quantum dynamics of the system can be described by the formally exact NakajimaZwanzig GQME, ${ }^{16,17,71-75,77}$ which assumes the following form under the conditions described earlier:

$$
\frac{d}{d t} \hat{\rho}_{s}(t)=-\frac{i}{\hbar} \mathcal{L}_{s} \hat{\rho}_{s}(t)-\int_{0}^{t} d \tau \mathcal{K}(\tau) \hat{\rho}_{s}(t-\tau) .
$$

Here, $\mathcal{L}_{s}(\cdot)=\left[\hat{H}_{s}, \cdot\right]$ and $\int_{0}^{t} d \tau \mathcal{K}(\tau) \hat{\rho}_{s}(t-\tau)$ represent the bath-free (Hamiltonian) and bath-induced (non-Hamiltonian) contributions to the system dynamics, respectively. The memory kernel, $\mathcal{K}(\tau)$, is explicitly given by either one of the following equivalent expressions:

$$
\begin{aligned}
\mathcal{K}(\tau) & =\frac{1}{\hbar^{2}} \operatorname{Tr}_{b}\left\{\mathcal{L}_{\mathrm{bs}} e^{-i \mathcal{Q} \mathcal{L} \tau / \hbar} \mathcal{Q} \mathcal{L}_{\mathrm{bs}} \hat{\rho}_{b}^{\mathrm{eq}}\right\} \\
& =\frac{1}{\hbar^{2}} \operatorname{Tr}_{b}\left\{\mathcal{L}_{\mathrm{bs}} e^{-i\left(\mathcal{L}-\mathcal{L}_{\mathrm{bs}} \mathcal{P}\right) \tau / \hbar} \mathcal{L}_{\mathrm{bs}} \hat{\rho}_{b}^{\mathrm{eq}}\right\},
\end{aligned}
$$

where $\mathcal{L}(\cdot)=[\hat{H}, \cdot], \mathcal{L}_{\mathrm{bs}}(\cdot)=\left[\hat{H}_{\mathrm{bs}}, \cdot\right], \mathcal{Q}=1-\mathcal{P}$ and

$$
\mathcal{P}(\cdot)=\hat{\rho}_{b}^{\mathrm{eq}} \otimes \operatorname{Tr}_{b}(\cdot) .
$$

As was shown in Ref. 78, the memory kernel, $\mathcal{K}(\tau)$, can be obtained from the following equation: 


$$
\mathcal{K}(\tau)=\mathcal{K}_{1}(\tau)+i \int_{0}^{\tau} d \tau^{\prime} \mathcal{K}_{1}\left(\tau-\tau^{\prime}\right) \mathcal{K}_{2}\left(\tau^{\prime}\right)
$$

where

$$
\mathcal{K}_{1}(\tau)=\frac{1}{\hbar^{2}} \operatorname{Tr}_{b}\left\{\mathcal{L}_{\mathrm{bs}} e^{-i \mathcal{L} \tau / \hbar} \mathcal{L}_{\mathrm{bs}} \hat{\rho}_{b}^{\mathrm{eq}}\right\} .
$$

$\mathcal{K}_{2}(\tau)$ is an auxiliary quantity which can be obtained by solving the following integral equation:

$$
\mathcal{K}_{2}(\tau)=\mathcal{K}_{3}(\tau)+i \int_{0}^{\tau} d \tau^{\prime} \mathcal{K}_{3}\left(\tau-\tau^{\prime}\right) \mathcal{K}_{2}\left(\tau^{\prime}\right),
$$

where

$$
\mathcal{K}_{3}(\tau)=\frac{1}{\hbar} \operatorname{Tr}_{b}\left\{e^{-i \mathcal{L} \tau / \hbar} \mathcal{L}_{\mathrm{bs}} \hat{\rho}_{b}^{\mathrm{eq}}\right\} .
$$

Thus, finding $\mathcal{K}(\tau)$ translates into calculating $\mathcal{K}_{1}(\tau)$ and $\mathcal{K}_{3}(\tau)$, followed by solving Eq. (11) for $\mathcal{K}_{2}(\tau)$, and Eq. (9) for $\mathcal{K}(\tau)$.

$\mathcal{K}_{1}(\tau)$ and $\mathcal{K}_{3}(\tau)$ are system superoperators. Within the framework of a Liouville-space-based description and tetradic notation, ${ }^{12}$ those superoperators can be represented by $N^{2} \times N^{2}$ matrices, in the case of an $N$-state system. We assume that $\hat{H}_{\mathrm{bs}}$ is given in terms of the system coordinates, such that $\hat{H}_{\mathrm{bs}}=\hat{H}_{\mathrm{bs}}(\hat{x})$, where $\hat{x}$ is the system position operator. The matrix elements of $\mathcal{K}_{1}(\tau)$ and $\mathcal{K}_{3}(\tau)$ in terms of the system position representation are then given by

$$
\begin{aligned}
\left\langle\left\langle x_{a}, x_{b}\left|\mathcal{K}_{1}(\tau)\right| x_{1}, x_{2}\right\rangle\right\rangle \\
=\frac{1}{\hbar^{2}}\left\{\left\langle\left[\hat{H}_{\mathrm{bs}}\left(x_{a}\right)-\hat{H}_{\mathrm{bs}}\left(x_{b}\right)\right](2, b, a, 1 ; \tau) \hat{H}_{\mathrm{bs}}\left(x_{1}\right)\right\rangle_{\mathrm{eq}}^{0}\right. \\
\left.\quad-\left\langle\hat{H}_{\mathrm{bs}}\left(x_{2}\right)\left[\hat{H}_{\mathrm{bs}}\left(x_{a}\right)-\hat{H}_{\mathrm{bs}}\left(x_{b}\right)\right](2, b, a, 1 ; \tau)\right\rangle_{\mathrm{eq}}^{0}\right\}
\end{aligned}
$$

and

$$
\begin{aligned}
\left\langle\left\langle x_{a}, x_{b}\left|\mathcal{K}_{3}(\tau)\right| x_{1}, x_{2}\right\rangle\right\rangle= & \frac{1}{\hbar}\left\{\left\langle\hat{I}_{b}(2, b, a, 1 ; \tau) \hat{H}_{\mathrm{bs}}\left(x_{1}\right)\right\rangle_{\mathrm{eq}}^{0}\right. \\
& \left.-\left\langle\hat{H}_{\mathrm{bs}}\left(x_{2}\right) \hat{I}_{b}(2, b, a, 1 ; \tau)\right\rangle_{\mathrm{eq}}^{0}\right\}
\end{aligned}
$$

where, the average $\langle\cdots\rangle_{\text {eq }}^{0}$ is defined similarly to that in Eq. (5), and

$$
\hat{\Gamma}(2, b, a, 1 ; \tau)=\left\langle x_{2}\left|e^{i \hat{H} \tau / \hbar}\right| x_{b}\right\rangle \hat{\Gamma}\left\langle x_{a}\left|e^{-i \hat{H} \tau / \hbar}\right| x_{1}\right\rangle .
$$

Here, $\hat{\Gamma}$ is a bath operator, which in our case is given in terms of either $\hat{H}_{\mathrm{bs}}(x)$ or $\hat{I}_{b}$ (the latter is the bath unity operator). Thus, all the information needed for determining the influence of the bath on the system is now contained in quantities of the form $\langle\hat{\Gamma}(2, b, a, 1 ; \tau) \hat{\Lambda}\rangle_{\text {eq }}^{0}$ where $\hat{\Gamma}$ and $\hat{\Lambda}$ are bath operator (and which depend parametrically on the system states). We refer to quantities of the form $\langle\hat{\Gamma}(2, b, a, 1 ; \tau) \hat{\Lambda}\rangle_{\mathrm{eq}}^{0}$ as SDBCFs.

In the case of an $N$-state system, the superoperators $\mathcal{K}(\tau)$, $\mathcal{K}_{1}(\tau)$, and $\mathcal{K}_{3}(\tau)$ are represented by $N^{2} \times N^{2}$ matrices. The need for computing $\sim N^{4}$ SDBCFs obviously imposes restrictions on the type of applications that will be accessible to the proposed approach. However, a significant number of nontrivial applications involve systems which can be described in terms of a relatively small number of states, where the computational effort would be manageable. Furthermore, the effective number of elements can be brought down somewhat, to $N^{3}(N-1) / 2$ in the case of $\mathcal{K}(\tau)$ and $\mathcal{K}_{1}(\tau)$, and $N^{2}\left(N^{2}+1\right) / 2$ in the case of $\mathcal{K}_{3}(\tau)$, by taking advantage of their symmetries. ${ }^{78}$

\section{A LSC APPROXIMATION OF THE SYSTEM- DEPENDENT BATH CORRELATION FUNCTIONS}

In the present section, we consider the application of the following approximation in the calculation of the SDBCFs:

$$
\begin{aligned}
& \operatorname{Tr}\left(\hat{A} e^{i \hat{H} t / \hbar} \hat{B} e^{-i \hat{H} t / \hbar}\right) \\
& \quad \approx \frac{1}{(2 \pi \hbar)^{f}} \int \mathbf{d q}_{0} \int \mathbf{d p}_{0} A_{W}\left(\mathbf{q}_{0}, \mathbf{p}_{0}\right) B_{W}\left(\mathbf{q}_{t}^{(\mathrm{Cl})}, \mathbf{p}_{t}^{(\mathrm{Cl})}\right) .
\end{aligned}
$$

Here, $f$ is the overall number of DOF, $\mathbf{q}=\left[q^{(1)}, \ldots, q^{(f)}\right]$ and $\mathbf{p}=\left[p^{(1)}, \ldots, p^{(f)}\right]$ are the corresponding coordinates and momenta

$$
A_{W}(\mathbf{q}, \mathbf{p})=\int \mathbf{d} \boldsymbol{\Delta} e^{-i \mathbf{p} \Delta / \hbar}\langle\mathbf{q}+\boldsymbol{\Delta} / 2|\hat{A}| \mathbf{q}-\mathbf{\Delta} / 2\rangle
$$

is the classical-like Wigner transform of the quantum mechanical operator $\hat{A}\left\{\text { with } \Delta=\left[\Delta^{(1)}, \ldots, \Delta^{(f)}\right]\right\}^{134,135}$ and $\mathbf{q}_{t}^{(\mathrm{Cl})}=\mathbf{q}_{t}^{(\mathrm{Cl})}\left(\mathbf{q}_{0}, \mathbf{p}_{0}\right)$ and $\mathbf{p}_{t}^{(\mathrm{Cl})}=\mathbf{p}_{t}^{(\mathrm{Cl})}\left(\mathbf{q}_{0}, \mathbf{p}_{0}\right)$ are propagated classically with the initial conditions $\mathbf{q}_{0}$ and $\mathbf{p}_{0}$. For later reference, we denote the approximation in Eq. (16) as the LSC approximation (the reason behind the name is clarified later).

The LSC approximation, as well as other related treatments which are based on the Wigner representation, have been considered, in a variety of contexts, by many workers in the past. ${ }^{116,117,125,130,134-152}$ For example, Eq. (16) can be obtained from the general theory of Wigner distributions via the following straightforward procedure:

$$
\begin{aligned}
& \operatorname{Tr}\left(e^{-\beta \hat{H}} e^{i \hat{H} t / \hbar} \hat{B} e^{-i \hat{H} t / \hbar} \hat{A}\right) \\
&=\frac{1}{(2 \pi \hbar)^{f}} \int \mathbf{d q}_{0} \int \mathbf{d p}_{0}\left[\hat{A} e^{-\beta \hat{H}}\right]_{W}\left(\mathbf{q}_{0}, \mathbf{p}_{0}\right) \\
& \times\left[e^{i \hat{H} t / \hbar} \hat{B} e^{-i \hat{H} t / \hbar}\right]_{W}\left(\mathbf{q}_{0}, \mathbf{p}_{0}\right) \\
& \approx \frac{1}{(2 \pi \hbar)^{f}} \int \mathbf{d q}_{0} \int \mathbf{d p}_{0}\left[\hat{A} e^{-\beta \hat{H}}\right]_{W}\left(\mathbf{q}_{0}, \mathbf{p}_{0}\right) B_{W} \\
& \times\left[\mathbf{q}_{t}^{(\mathrm{Cl})}, \mathbf{p}_{t}^{(\mathrm{Cl})}\right] .
\end{aligned}
$$

The first equality in Eq. (18) is exact, and the second is based on the $\hbar \rightarrow 0$ limit of the equation of motion of $\left[e^{i \hat{H} t / \hbar} \hat{B} e^{-i \hat{H} t / \hbar}\right]_{W}$. The LSC approximation can also be derived within the framework of the semiclassical initial-valuerepresentation methodology. ${ }^{116,130,132,147-151}$ For example, Miller and co-workers, have recently derived it by linearizing the forward-backward action in the semiclassical initialvalue-representation (IVR) expression for a quantum- 
mechanical $\mathrm{CF}$, with respect to the difference between the forward and backward trajectories (the LSC approximation has been denoted LSC-IVR by those workers). We also note that the very same approximation can be derived by linearizing the forward-backward action in the exact real-time path integral expression for the $\mathrm{CF}$, and without explicitly invoking the semiclassical initial-value-representation approximation. ${ }^{153}$

The major advantage of a LSC-based approach has to do with its computational feasibility (although the computation of the Wigner transform in systems with many DOF is not trivial). ${ }^{116,154-156}$ The approximation also has the attractive features of being exact at the initial time, at the classical limit, and for harmonic systems. Its main disadvantage has to do with the fact that it can only capture quantum dynamical effects that arise from short-time interferences between the various trajectories (the longer time dynamics is purely classical). ${ }^{148}$ However, it should be noted that this may represent less of a problem in the case of condensed phase systems, where CFs are relatively short-lived.

It should be noted that the LSC approximation can also be used to describe the nonequilibrium dynamics of a system coupled to a bath. ${ }^{148,150}$ The procedure is based on the fact that the matrix elements of $\hat{\rho}_{s}(t)$, in a representation of one's choice, can be written in the form of CFs of the overall system [cf. Eq. (16)]:

$$
\begin{aligned}
\left\langle u\left|\hat{\rho}_{s}(t)\right| v\right\rangle= & \operatorname{Tr}_{s}\left[|v\rangle\langle u| \hat{\rho}_{s}(t)\right]=\operatorname{Tr}[|v\rangle\langle u| \hat{\rho}(t)] \\
= & \operatorname{Tr}\left[\hat{\rho}(0) e^{i \hat{H} t / \hbar}|v\rangle\langle u| e^{-i \hat{H} t / \hbar}\right] \\
\approx & \frac{1}{(2 \pi \hbar)^{f}} \int \mathbf{d q}_{0} \int \mathbf{d p}_{0}[\hat{\rho}(0)]_{W} \\
& \times\left(\mathbf{q}_{0}, \mathbf{p}_{0}\right)[|v\rangle\langle u|]_{W}\left(\mathbf{q}_{t}^{(\mathrm{Cl})}, \mathbf{p}_{t}^{(\mathrm{Cl})}\right] .
\end{aligned}
$$

However, it is important to note that the reliability of the approximation in the last equality of Eq. (19) is limited to very short times.

Employing the LSC approximation, Eq. (16), for calculating the SDBCFs is straightforward once the latter are rewritten as CFs of the overall system

$$
\begin{aligned}
\langle\hat{\Gamma}(2, b, a, 1 ; \tau) \hat{\Lambda}\rangle_{\mathrm{eq}}^{0} & \\
= & \operatorname{Tr}_{b}\left\{\hat{\Lambda} \hat{\rho}_{b}^{\mathrm{eq}}\left\langle x_{2}\left|e^{i \hat{H} \tau / \hbar}\right| x_{b}\right\rangle \hat{\Gamma}\left\langle x_{a}\left|e^{-i \hat{H} \tau / \hbar}\right| x_{1}\right\rangle\right\} \\
= & \operatorname{Tr}\left\{\hat{\Lambda} \hat{\rho}_{b}^{\mathrm{eq}}\left|x_{1}\right\rangle\left\langle x_{2}\left|e^{i \hat{H} \tau / \hbar}\right| x_{b}\right\rangle\left\langle x_{a}\right| \hat{\Gamma} e^{-i \hat{H} \tau / \hbar}\right\} \\
\approx & \frac{1}{(2 \pi \hbar)^{N+1}} \int \mathbf{d} \mathbf{Q}_{0} \int \mathbf{d} \mathbf{P}_{0} \int d x_{0} \int d p_{0}\left[\hat{\Lambda} \hat{\rho}_{b}^{\mathrm{eq}}\right]_{W} \\
& \times\left(\mathbf{Q}_{0}, \mathbf{P}_{0}\right)\left[\left|x_{1}\right\rangle\left\langle x_{2}\right|\right]_{W}\left(x_{0}, p_{0}\right) \Gamma_{W}\left[\mathbf{Q}_{t}^{(\mathrm{Cl})}, \mathbf{P}_{t}^{(\mathrm{Cl})}\right]\left[\left|x_{b}\right\rangle\right. \\
& \left.\times\left\langle x_{a}\right|\right]_{W}\left[x_{t}^{(\mathrm{Cl})}, p_{t}^{(\mathrm{Cl})}\right],
\end{aligned}
$$

where $\mathbf{Q}=\left[Q^{(1)}, \ldots, Q^{(N)}\right]$ and $\mathbf{P}=\left[P^{(1)}, \ldots, P^{(N)}\right]$ correspond to the coordinates and conjugate momenta of the bath DOF, while $x$ and $p$ correspond to the coordinate and conjugate momentum of the system DOF (assumed to be one dimensional for the sake of simplicity).
In many applications of interest, such as nonadiabatic electron transfer and optical spectroscopy, the system corresponds to electronic DOF, and is most conveniently described in terms of a discrete manifold of states, rather than in terms of the electronic coordinates and momenta. In such cases, it is convenient to employ the Meyer-Miller (MM) method for mapping the discrete electronic manifold onto an isomorphic system that consists of harmonic modes. ${ }^{132,148,150,157-162}$ More specifically, if the system Hilbert space is spanned by the discrete basis $\{|1\rangle, \ldots,|n\rangle\}$, than, following Refs. 161 and 162, one can represent the corresponding eigen-projectors in terms of bosonic creation and annihilation operators

$$
|j\rangle\langle k| \leftrightarrow \hat{a}_{j}^{\dagger} \hat{a}_{k}, \quad\left[\hat{a}_{j}^{\dagger}, \hat{a}_{k}\right]=\delta(j, k) .
$$

The mapping in Eq. (21) is justified by the fact that the operators $\{|j\rangle\langle k|\}$ satisfy the same commutation relations as the operators $\left\{\hat{a}_{j}^{\dagger} \hat{a}_{k}\right\}$. Furthermore, the operators $\hat{a}_{j}^{\dagger}$ and $\hat{a}_{j}$ can be associated with a fictitious harmonic mode which corresponds to the following coordinate and momentum operators

$\hat{q}_{j}=\sqrt{\frac{\hbar}{2}}\left(\hat{a}_{j}+\hat{a}_{j}^{\dagger}\right), \quad \hat{p}_{j}=-i \sqrt{\frac{\hbar}{2}}\left(\hat{a}_{j}-\hat{a}_{j}^{\dagger}\right), \quad\left[\hat{q}_{j}, \hat{p}_{j}\right]=i \hbar$.

As a result, one can map the original discrete states onto the corresponding states of $n$ harmonic modes

$$
|1\rangle \leftrightarrow|1,0, \ldots, 0\rangle, \quad|2\rangle \leftrightarrow|0,1, \ldots, 0\rangle, \ldots,|n\rangle \leftrightarrow|0,0, \ldots, 1\rangle .
$$

One can also map the operators defined within the original discrete manifold of electronic states, onto operators that can be given in terms of the coordinates and momenta operators of the harmonic modes

$$
\begin{aligned}
& |j\rangle\langle j| \leftrightarrow \hat{a}_{j}^{\dagger} \hat{a}_{j}=\frac{1}{2 \hbar}\left(\hat{q}_{j}^{2}+\hat{p}_{j}^{2}-\hbar\right), \\
& |j\rangle\langle k| \leftrightarrow \hat{a}_{j}^{\dagger} \hat{a}_{k}=\frac{1}{2 \hbar}\left(\hat{q}_{j} \hat{q}_{k}+\hat{p}_{j} \hat{p}_{k}+i \hat{q}_{j} \hat{p}_{k}-i \hat{p}_{j} \hat{q}_{k}\right) .
\end{aligned}
$$

Finally, as long as the Hamiltonian only includes operators of the form $\hat{a}_{j}^{\dagger} \hat{a}_{k}$, as it must, we are assured that the dynamics will be restricted to the subspace of the Hilbert space of the harmonic modes, which is spanned by the states in Eq. (23).

It is instructive to consider the application of the LSC method to the calculation of the SDBCFs in the relatively simple case of a two-level system (TLS) $(n=2)$. To this end, we consider the following general Hamiltonian that describes a TLS coupled to a bath

$$
\hat{H}=\Omega \hat{\sigma}_{x}+\Delta \hat{\sigma}_{z}+\sum_{l=1}^{N} \frac{\left[P^{(l)}\right]^{2}}{2 M^{(l)}}+V(\hat{\mathbf{Q}})+\Lambda(\hat{\mathbf{Q}}) \hat{\sigma}_{z} .
$$

Here, $\quad \hat{\sigma}_{x}=|+\rangle\langle-|+|-\rangle\langle+|, \quad \hat{\sigma}_{y}=(|+\rangle\langle-|-|-\rangle\langle+|) / i$ and $\hat{\sigma}_{z}=|+\rangle\langle+|-|-\rangle\langle-|$, where $\hat{\sigma}_{z}| \pm\rangle= \pm| \pm\rangle$. It should be noted that in this case, $\hat{\sigma}_{z}$ plays the role of the system coordinate, $\hat{x}$. Following Refs. 150, 161, and 162, the TLS is then mapped onto the isomorphic system that consists of two harmonic oscillators with coordinates and momenta $\left(q_{+}, p_{+}\right)$and $\left(q_{-}, p_{-}\right)$, respectively, such that $|+\rangle \leftrightarrow|1,0\rangle$ 
and $|-\rangle \leftrightarrow|0,1\rangle$. The LSC approximation of the SDBCF in Eq. (20) involves the following four system Wigner transforms that correspond to the operators $|+\rangle\langle+||-\rangle,\langle-|$, $|+\rangle\langle-|$ and $|-\rangle\langle+|$, respectively,

$$
\begin{aligned}
{[|1,0\rangle\langle 1,0|]_{W}(\mathbf{q}, \mathbf{p})=} & \frac{2^{3}}{\hbar}\left(q_{+}^{2}+p_{+}^{2}-\frac{\hbar}{2}\right) \\
& \times e^{-\left(q_{+}^{2}+p_{+}^{2}+q_{-}^{2}+p_{-}^{2}\right) / \hbar}, \\
{[|0,1\rangle\langle 0,1|]_{W}(\mathbf{q}, \mathbf{p})=} & \frac{2^{3}}{\hbar}\left(q_{-}^{2}+p_{-}^{2}-\frac{\hbar}{2}\right) \\
{[|1,0\rangle\langle 0,1|]_{W}(\mathbf{q}, \mathbf{p})=} & \frac{2^{3}}{\hbar}\left(q_{-}^{-\left(q_{+}^{2}+p_{+}^{2}+q_{-}^{2}+p_{-}^{2}\right) / \hbar},\right. \\
& \times e^{-\left(q_{+}^{2}+p_{+}^{2}+q_{-}^{2}+p_{-}^{2}\right) / \hbar} \\
{[|0,1\rangle\langle 1,0|]_{W}(\mathbf{q}, \mathbf{p})=} & \frac{2^{3}}{\hbar}\left(q_{-}^{-i p_{+}}\right)\left(q_{+}+i p_{+}\right) \\
& \times e^{-\left(q_{+}^{2}+p_{+}^{2}+q_{-}^{2}+p_{-}^{2}\right) / \hbar} .
\end{aligned}
$$

Finally, we note that, within the LSC approximation, $q_{ \pm}(t), p_{ \pm}(t), \mathbf{Q}(t)$, and $\mathbf{P}(t)$ are propagated according to classical mechanics, subject to the Hamiltonian in Eq. (25). In practice, it is convenient to propagate the quantities $\sigma_{x}^{\mathrm{MM}}=\left(q_{+} q_{-}+p_{+} p_{-}\right) / \hbar, \quad \sigma_{y}^{\mathrm{MM}}=\left(q_{+} p_{-}-q_{-} p_{+}\right) / \hbar$, and $\sigma_{z}^{\mathrm{MM}}=\left(q_{+}^{2}+p_{+}^{2}-q_{-}^{2}-p_{-}^{2}\right) / 2 \hbar$, which are the classical variables that correspond to the operators $\hat{\sigma}_{x}, \hat{\sigma}_{y}$, and $\hat{\sigma}_{z}$, within the MM mapping. The equations of motion for $\sigma_{x}^{\mathrm{MM}}$, $\sigma_{y}^{\mathrm{MM}}, \sigma_{z}^{\mathrm{MM}}, \mathbf{Q}(t)$, and $\mathbf{P}(t)$ are given by

$$
\begin{aligned}
& \dot{\sigma}_{x}^{\mathrm{MM}}=-\frac{2}{\hbar}[\Delta+\Lambda(\mathbf{Q})] \sigma_{y}^{\mathrm{MM}}, \\
& \dot{\sigma}_{y}^{\mathrm{MM}}=-\frac{2}{\hbar} \Omega \sigma_{z}^{\mathrm{MM}}+\frac{2}{\hbar}[\Delta+\Lambda(\mathbf{Q})] \sigma_{x}^{\mathrm{MM}}, \\
& \dot{\sigma}_{z}^{\mathrm{MM}}=\frac{2}{\hbar} \Omega \sigma_{y}^{\mathrm{MM}}, \\
& \dot{Q}^{(k)}=\frac{P^{(k)}}{M^{(k)}}, \\
& \dot{P}^{(k)}=-\frac{\partial V(\mathbf{Q})}{\partial Q^{(k)}}-\frac{\partial \Lambda(\mathbf{Q})}{\partial Q^{(k)}} \sigma_{z}^{\mathrm{MM}} .
\end{aligned}
$$

\section{ILLUSTRATIVE APPLICATIONS}

In this section we employ the LSC approximation and MM mapping, in order to calculate the SDBCFs, memory kernel superoperator, $\mathcal{K}(\tau)$, and subsequently simulate the reduced quantum dynamics, in the case of two nontrivial model systems. Both examples involve a TLS coupled to a bath, with the overall Hamiltonian as in Eq. (25). The first example demonstrates the accuracy of the methodology, in the context of a benchmark spin-boson problem, ${ }^{163,164}$ while the second example tests the feasibility of applying the method to anharmonic systems.

\section{A. A two-level system linearly coupled to a harmonic bath}

Consider an overall system whose Hamiltonian has the form of Eq. (25), with

$$
V(\mathbf{Q})=\sum_{j=1}^{N} \frac{1}{2} M^{(j)}\left[\omega^{(j)}\right]^{2}\left[\hat{Q}^{(j)}\right]^{2},
$$

and

$$
\Lambda(\hat{\mathbf{Q}})=-\sum_{j=1}^{N} c^{(j)} \hat{Q}^{(j)} .
$$

A complete characterization of this harmonic bath is provided by its spectral density function

$$
J(\omega)=\frac{\pi}{2} \sum_{j} \frac{\left[c^{(j)}\right]^{2}}{M^{(j)} \omega^{(j)}} \delta\left[\omega-\omega^{(j)}\right] .
$$

The Wigner transform of the bath operator $\Lambda(\hat{\mathbf{Q}})$ is trivial and given by $[\Lambda(\hat{\mathbf{Q}})]_{W}=\Lambda(\mathbf{Q})$. The Wigner transform of $\Lambda(\hat{\mathbf{Q}}) \hat{\rho}_{b}^{\text {eq }}$ only involves Gaussian integrals and can therefore be evaluated analytically

$$
\begin{aligned}
{\left[\Lambda(\hat{\mathbf{Q}}) \hat{\rho}_{b}^{\mathrm{eq}}\right]_{W}(\mathbf{Q}, \mathbf{P}) } & =2^{N}\left\{\prod_{j=1}^{N} \tanh \left[\beta \hbar \omega^{(j)} / 2\right]\right\} \\
& \times \exp \left(-\sum_{j=1}^{N} \frac{\tanh \left[\beta \hbar \omega^{(j)} / 2\right]}{\hbar \omega^{(j)} / 2}\left\{\frac{\left[\hat{P}^{(j)}\right]^{2}}{2 M^{(j)}}+\frac{1}{2} M^{(j)}\right.\right. \\
& \left.\left.\times\left[\omega^{(j)}\right]^{2}\left[\hat{Q}^{(j)}\right]^{2}\right\}\right) \\
& \times \sum_{k=1}^{N} c^{(k)}\left\{Q^{(k)}-i \frac{\tanh \left[\beta \hbar \omega^{(k)} / 2\right]}{M^{(k)} \omega^{(k)}} P^{(k)}\right\} .
\end{aligned}
$$

The results reported later were obtained for a spectral density of the form

$$
J(\omega)=\xi \frac{\pi}{2} \omega e^{-\omega / \omega_{c}}
$$

and the following values of the various parameters: $\Delta=\Omega$ $=1.0, \beta \hbar \Omega=5.0, \xi=0.1$, and $\omega_{c} / \Omega=7.5$. It should be noted that results based on numerically exact SDBCFs have been reported for the same model and parameters in Ref. 78. In the present paper, we present results obtained by using the LSC approximation and MM mapping in order to compute the SDBCFs. It should be noted that the overall Hamiltonian, including the TLS in the MM representation, is clearly anharmonic, due to the term $\Lambda(\hat{\mathbf{Q}}) \hat{\sigma}_{z} \leftrightarrow-\sum_{j=1}^{N} c^{(j)} \hat{Q}^{(j)}\left(q_{+}^{2}\right.$ $\left.+p_{+}^{2}-q_{-}^{2}-p_{-}^{2}\right) / 2 \hbar$. Thus, the LSC method is not formally exact in this case.

The procedure for obtaining the memory kernel, and subsequently simulating the system dynamics, is similar to that followed in Ref. 78. Briefly, the SDBCFs are computed and used in order to calculate $\mathcal{K}_{1}(\tau)$ and $\mathcal{K}_{3}(\tau)$ on a 300 point time grid with a time step of $0.01 \Omega^{-1}$. Equation (11) is then solved for $\mathcal{K}_{2}(\tau)$, via an iterative procedure, with 


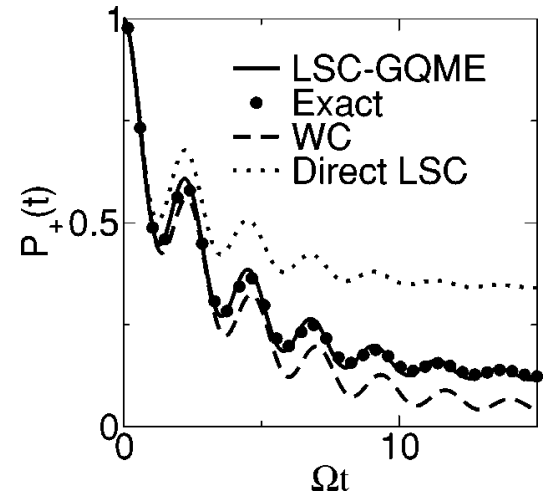

FIG. 2. The relaxation of $P_{+}(t)=\left\langle+\left|\hat{\rho}_{s}(t)\right|+\right\rangle$ to equilibrium in the case of a TLS linearly coupled to a harmonic bath. Shown are the predictions of the GQME with LSC-approximated kernel (LSC-GQME, solid line), the exact result (solid circles), a prediction based on the WC treatment (dashed line), and the prediction based on direct application of LSC (dotted line).

$\mathcal{K}_{2}(\tau)=\mathcal{K}_{3}(\tau)$ as the initial guess (10-20 iterations were required for convergence). The resulting $\mathcal{K}_{2}(\tau)$ is then substituted into Eq. (9), so as to obtain $\mathcal{K}(\tau)$ on the same 300 point time grid. The matrix representing $\mathcal{K}(\tau)$ is kept in memory as a $2 \times 2 \times 300$ array throughout the subsequent numerical solution of the GQME, which is carried out by the second-order Runge-Kutta method. ${ }^{165}$ A time step of 0.01 $\Omega^{-1}$ has been used, and $\hat{\rho}_{s}(t)$ over the previous 300 steps has been kept in memory, as a $2 \times 2 \times 300$ array, in order to evaluate the non-Markovian contribution to the time derivative.

In Fig. 2, we show the relaxation of the population of state $|+\rangle, \quad P_{+}(t)=\left\langle+\left|\hat{\rho}_{s}(t)\right|+\right\rangle$, to equilibrium, starting from the initial state $\hat{\rho}_{s}(0)=|+\rangle\langle+|$, as obtained via the LSC-GQME method, where one solves the GQME with a kernel that was computed based on the LSC approximation and MM mapping (solid line). Also shown in this figure are: (1) The numerically exact result, as obtained via the iterative tensor quasi-adiabatic propagator method of Makri et al. (solid circles) $;^{78}$ (2) The result based on the WC treatment ${ }^{78}$ (dashed line); and (3) The result obtained via a direct application of the LSC approximation to the TLS dynamics, Eq. (19) (dotted line). It is first interesting to compare the results of the WC and direct LSC treatments. Both are only accurate at very short times. In the case of the direct LSC treatment, the inaccuracy at longer times can be traced back to the fact that the dynamics is purely classical. This is manifested by the fact that the coherent oscillations are overdamped and the asymptotic equilibrium state is classical. In the case of the WC treatment, the inaccuracy results from neglecting higher order terms in the system-bath coupling. Thus, the coherent oscillations are underdamped, since the bath-induced decoherence is underestimated. Furthermore, although the asymptotic state corresponds to a quantum-mechanical equilibrium, the latter is described by a system density operator

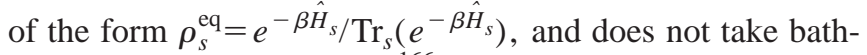
induced shifts into account. ${ }^{166}$

The exact result in Fig. 2 lies between the predictions of the direct LSC and WC treatments. The damping of the coherent oscillations is weaker in comparison to the direct LSC treatment, but stronger in comparison to the WC treatment.

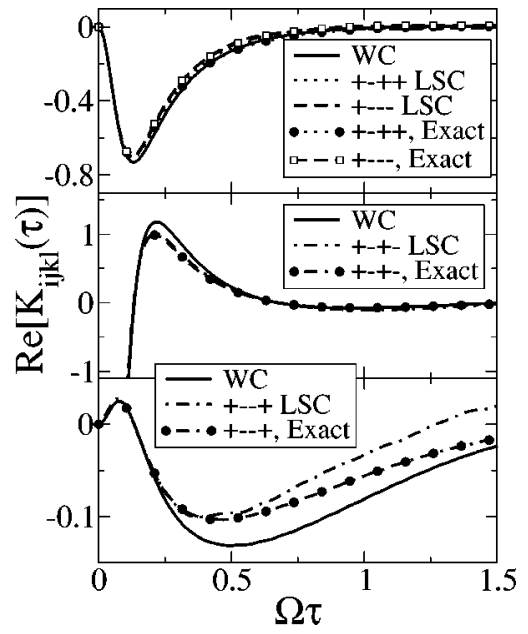

FIG. 3. The exact, LSC-based and WC-based real parts of $\langle\langle+-|\mathcal{K}(\tau)|++\rangle$ and $\langle\langle+-|\mathcal{K}(\tau)|--\rangle\rangle$ (upper panel), $\langle\langle+-|\mathcal{K}(\tau)|+-\rangle$ (middle panel), and $\langle\langle+|\mathcal{K}(\tau)|-+\rangle\rangle$ (lower panel), for a TLS coupled to a harmonic bath. Note that $\langle\langle+-|\mathcal{K}(\tau)|++\rangle\rangle=\langle\langle+-|\mathcal{K}(\tau)|--\rangle\rangle$ at the WC limit.

Furthermore, the asymptotic equilibrium state clearly deviates from the corresponding classical equilibrium state, and is also affected by the coupling to the bath, which is evident from the significant deviation relative to the asymptotic state that the WC treatment gives rise to. Finally, and most importantly, Fig. 2 shows that restricting the use of LSC to the calculation of the short-lived memory kernel leads to a very accurate result, which is almost indistinguishable from the exact result. Thus, the LSC-GQME methodology is found to be very accurate in a case where both the direct LSC and WC treatments fail.

In the case of a TLS, there are only four independent matrix elements of the memory kernel, which can be chosen as $^{78}\langle\langle+-|\mathcal{K}(\tau)|++\rangle\rangle,\langle\langle+-|\mathcal{K}(\tau)|--\rangle\rangle,\langle\langle+-|\mathcal{K}(\tau)|+-\rangle\rangle$, and $\langle\langle+-|\mathcal{K}(\tau)|-+\rangle\rangle$. Their real and imaginary parts, as obtained from the exact, LSC-based and WC treatments, are shown in Figs. 3 and 4, respectively. The agreement of the LSC-based and exact results is much better than the agreement of the WC results with them. At the same time, the agreement between the LSC-based and exact results is clearly not as good as in the case of the actual system dy-

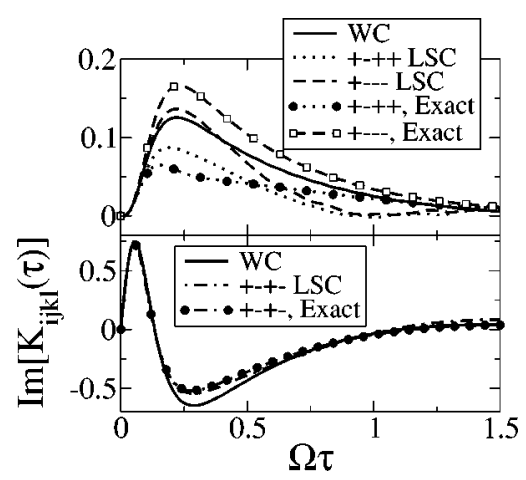

FIG. 4. The exact, LSC-based and WC-based imaginary parts of $\langle\langle+-|\mathcal{K}(\tau)|$ $++\rangle$ and $\langle\langle+-|\mathcal{K}(\tau)|--\rangle\rangle$ (upper panel), and $\langle\langle+-|\mathcal{K}(\tau)|+-\rangle$ (lower panel), for a TLS coupled to a harmonic bath. Note that $\operatorname{Im}\langle\langle+-|\mathcal{K}(\tau)|$ $-+\rangle\rangle=0$ in this case (see lower panel of Fig. 3 for its real part). Note that $\langle\langle+-|\mathcal{K}(\tau)|++\rangle=\langle\langle+-|\mathcal{K}(\tau)|--\rangle$ at the WC limit. 


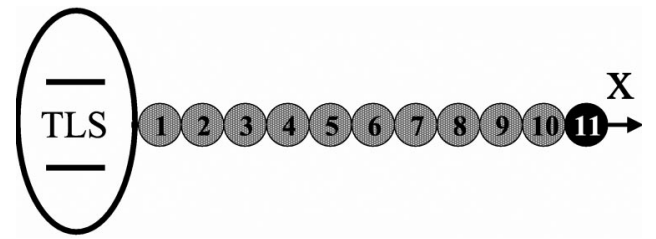

FIG. 5. A schematic view of the helium chain model. The TLS is directly coupled to helium atom 1, and the interaction potential depends on the TLS state. The helium atoms interact via nearest neighbor Lennard-Jones potentials. Helium atoms $1-10$ are free to move, while the 11 th helium atom is held fixed.

namics (see Fig. 2). We view this observation as encouraging from a computational point of view, since it suggests that the actual system dynamics is not very sensitive to the fine details of the SDBCFs. Thus, a moderately accurate memory kernel may be sufficient for obtaining reliable predictions of the system relaxation dynamics.

\section{B. A two-level system nonlinearly coupled to an anharmonic bath}

Our second example involves the nonadiabatic relaxation dynamics of a TLS coupled to an anharmonic bath. The latter consists of a linear chain of 11 helium atoms, that lie along the $x$ axis (cf. Fig. 5). We assume that the TLS corresponds to an internal DOF of an "atom" A, which is held fixed at $x=0$. The TLS Hamiltonian is given by

$$
\hat{H}_{s}^{0}=\Omega \hat{\sigma}_{x}+\Delta \hat{\sigma}_{z} .
$$

The bath Hamiltonian is similar to that employed in Refs. 88, 97, 154, and 167. The instantaneous positions of the first ten helium atoms $(i=1,2, \ldots, 10)$ are given by $\left[x_{i}=\sigma_{\mathrm{He}-\mathrm{A}}+(i\right.$ -1) $\left.\sigma_{\mathrm{He}-\mathrm{He}}+\delta_{i}\right]$, and the 11th, and last, helium atom is held fixed at $x_{11}=\sigma_{\mathrm{He}-\mathrm{A}}+10 \sigma_{\mathrm{He}-\mathrm{He}}$ (cf. Fig. 5). The overall Hamiltonian is given by

$$
\hat{H}=\hat{H}_{s}^{0}+\sum_{k=1}^{10} \frac{\left[p^{(k)}\right]^{2}}{2 M_{\mathrm{He}}}+V_{+}(\hat{\mathbf{x}})|+\rangle\left\langle+\left|+V_{-}(\hat{\mathbf{x}})\right|-\right\rangle\langle-|,
$$

where $\hat{\mathbf{x}}=\left(\hat{x}^{(1)}, \ldots, \hat{x}^{(10)}\right)$ and $\hat{\mathbf{p}}=\left[\hat{p}^{(1)}, \ldots, \hat{p}^{(10)}\right]$ are the operators representing the coordinates and momenta of the Helium atoms, and

$$
\begin{aligned}
V_{ \pm}= & v_{\mathrm{LJ}}^{\mathrm{He}-\mathrm{A}}\left(\sigma_{\mathrm{He}-\mathrm{A}}+\delta_{1} \pm r_{0}\right) \\
& +\sum_{i=1}^{10} v_{\mathrm{LJ}}^{\mathrm{He}-\mathrm{He}}\left(\sigma_{\mathrm{He}-\mathrm{He}}+\delta_{i+1}-\delta_{i}\right) .
\end{aligned}
$$

Here,

$$
v_{\mathrm{LJ}}(r)=4 \epsilon\left[\left(\frac{\sigma}{r}\right)^{12}-\left(\frac{\sigma}{r}\right)^{6}\right],
$$

is the familiar Lennard-Jones (LJ) potential, with $\sigma$ and $\epsilon$ given by $\left\{\sigma_{\mathrm{He}-\mathrm{A}}, \epsilon_{\mathrm{He}-\mathrm{A}}\right\}$ and $\left\{\sigma_{\mathrm{He}-\mathrm{He}}, \epsilon_{\mathrm{He}-\mathrm{He}}\right\}$ for the $\mathrm{He}-\mathrm{A}$, and $\mathrm{He}-\mathrm{He}$ interactions, respectively. It should be noted that the potential energy in Eq. (35) only includes nearestneighbor interactions. Importantly, the interaction between atom $\mathrm{A}$ and the first helium atom depends on the internal state of atom A. More specifically, the equilibrium distance between atom $\mathrm{A}$ and the first helium atom is either $2{ }^{1 / 6} \sigma_{\mathrm{He}-\mathrm{A}}+r_{0}$ or $2^{1 / 6} \sigma_{\mathrm{He}-\mathrm{A}}-r_{0}$, depending on whether the internal state of atom A is given by $|+\rangle$ or $|-\rangle$. For example, this could correspond to a situation where the electronic wave function of the excited electronic state of the solute atom $\mathrm{A}$ is less spatially confined than that in the ground electronic state.

In the next step, we rewrite the Hamiltonian in Eq. (34) in the form of Eq. (2), such that the bath Hamiltonian is given by

$$
\hat{H}_{b}=\sum_{k=1}^{10} \frac{\left[p^{(k)}\right]^{2}}{2 M_{\mathrm{He}}}+\frac{1}{2}\left[V_{+}(\hat{\mathbf{x}})+V_{-}(\hat{\mathbf{x}})\right],
$$

the system Hamiltonian is given by

$$
\hat{H}_{s}=\Omega \hat{\sigma}_{x}+\left[\Delta+\frac{1}{2}\left\langle V_{+}(\hat{\mathbf{x}})-V_{-}(\hat{\mathbf{x}})\right\rangle_{\mathrm{eq}}^{0}\right] \hat{\sigma}_{z},
$$

and the system-bath coupling is given by

$$
\hat{H}_{\mathrm{bs}}=\hat{\Lambda}(\hat{\mathbf{x}}) \otimes \hat{\sigma}_{z},
$$

where

$$
\hat{\Lambda}=\frac{1}{2}\left[V_{+}(\hat{\mathbf{x}})-V_{-}(\hat{\mathbf{x}})-\left\langle V_{+}(\hat{\mathbf{x}})-V_{-}(\hat{\mathbf{x}})\right\rangle_{\mathrm{eq}}^{0}\right] .
$$

It should be noted that the term $\left\langle V_{+}(\hat{\mathbf{x}})+V_{-}(\hat{\mathbf{x}})\right\rangle_{\mathrm{eq}}^{0} \hat{\sigma}_{z} / 2$ has been added to the original system Hamiltonian, Eq. (33), such that $\hat{H}_{\text {bs }}$ satisfies Eq. (5).

Calculations pertaining to this model have been performed using the following values of the parameters: $T$ $=40 \mathrm{~K}, \quad \sigma_{\mathrm{He}-\mathrm{A}}=4.944$ a.u.,$\quad \sigma_{\mathrm{He}-\mathrm{He}}=4.310$ a.u.,$\quad \epsilon_{\mathrm{He}-\mathrm{A}} / k_{B}$ $=25.1 \mathrm{~K}, \quad \epsilon_{\mathrm{He}-\mathrm{He}} / k_{B}=10.2 \mathrm{~K}, \Omega=1.0 \times 10^{-4}$ a.u., $\Delta=1.2$ $\times 10^{-4}$ a.u., and $r_{0}=0.2$ a.u. Unlike in the case of the harmonic bath, the Wigner transform of $\hat{\Lambda} \hat{\rho}_{b}^{\text {eq }}$ cannot be calculated analytically for this anharmonic model. At the same time, a numerically exact calculation of the corresponding multidimensional integral via conventional Monte Carlo (MC) techniques is prohibitively expensive, due to the oscillatory phase factor, $e^{-i \mathbf{P}_{0} \Delta / \hbar}$. One way of overcoming this problem is by introducing an approximation that will allow us to perform the Wigner integral analytically. We have recently proposed such an approximation, which is based on a quadratic expansion, in terms of $\boldsymbol{\Delta}$, of the ratio $\left\langle\mathbf{Q}_{0}\right.$ $\left.+\boldsymbol{\Delta} / 2\left|e^{-\beta \hat{H}_{b}}\right| \mathbf{Q}_{0}-\boldsymbol{\Delta} / 2\right\rangle /\left\langle\mathbf{Q}_{0}\left|e^{-\beta \hat{H}_{b}}\right| \mathbf{Q}_{0}\right\rangle .{ }^{154}$ The resulting methodology will be referred to below as the local harmonic approximated LSC (LHA-LSC). The LHA-LSC methodology has several important advantages such as reproducing the correct classical and $t=0$ limits, as well as accounting for both quantum and anharmonic aspects of the bath when sampling its initial configurations. It has also been observed to lead to accurate results when applied to the challenging problem of calculating high-frequency vibrational energy relaxation rate constants (the reader is referred to Refs. 154 and 155 for further details). The results reported later are based on using the LHA-LSC method for calculating the Wigner transform of $\hat{\Lambda} \hat{\rho}_{b}^{\mathrm{eq}}$.

In Fig. 6, we show the real and imaginary parts of the free-bath CF, $C(t)=\langle\hat{\Lambda}(0) \hat{\Lambda}(t)\rangle$, as obtained from a LHALSC-based calculation, with $\hat{\Lambda}$ as in Eq. (40). This CF rep- 


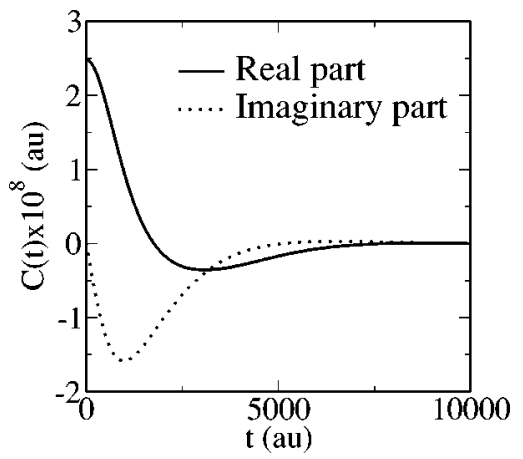

FIG. 6. The free bath $\operatorname{CF} C(t)=\langle\hat{\Lambda}(0) \hat{\Lambda}(t)\rangle$ for the helium chain model, as obtained via the LHA-LSC method.

resents the only input required in order to account for the influence of the bath on the system in the WC limit. It is important to note that this CF decays to zero and is relatively short lived, which justifies the view that a bath consisting of 11 helium atoms can already be considered as a condensedphase host.

In Fig. 7, we show the relaxation of the population of state $|+\rangle, P_{+}(t)=\left\langle+\left|\hat{\rho}_{s}(t)\right|+\right\rangle$, to equilibrium, starting from the initial state $\hat{\rho}_{s}(0)=|+\rangle\langle+|$, as obtained by solving the GQME with a kernel that was computed based on the LHA-LSC approximation (solid line). Also shown in this figure are: (1) The result obtained via a direct application of LHA-LSC to the TLS dynamics, Eq. (19) (dotted line); and (2) The result obtained via the WC treatment, with the freebath CF obtained via LHA-LSC (dashed line). It should be noted that a numerically exact quantum mechanical calculation is prohibitively expensive in this case, and is therefore not available for comparison. The following observations can be made based on Fig. 7:

- As expected, the results from all three methods coincide at very short times. The corresponding time scale is comparable to the lifetime of the CF in Fig. 6. This suggests that restricting the use of the LHA-LSC approximation to

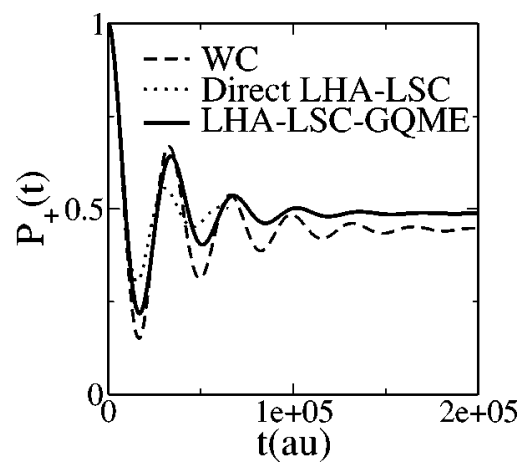

FIG. 7. The relaxation of $P_{+}(t)=\left\langle+\left|\hat{\rho}_{s}(t)\right|+\right\rangle$ to equilibrium in the case of a TLS nonlinearly coupled to an anharmonic bath which consists of a chain of eleven helium atoms. Shown are the predictions of the GQME with a LHA-LSC approximated kernel (LHA-LSC-GQME, solid line), a prediction based on the WC treatment (dashed line), and the prediction based on direct application of LHA-LSC (dotted line). The latter is only given at short times due to an increase in the number of unstable trajectories that the MM mapping gives rise to.

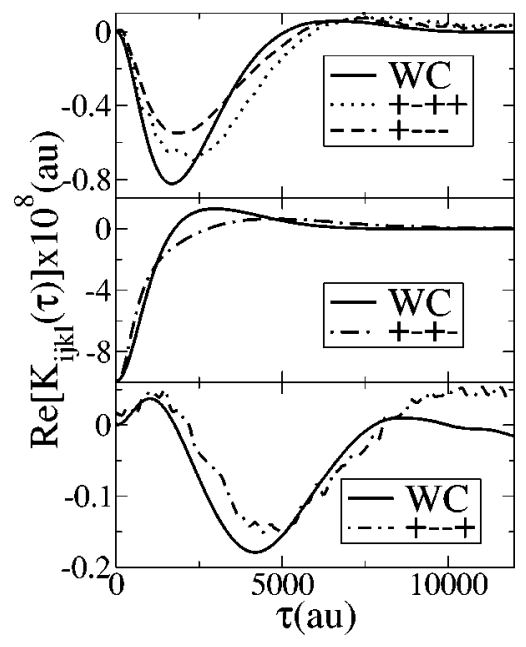

FIG. 8. The LHA-LSC-based and WC-based real parts of $\langle\langle+-|\mathcal{K}(\tau)|++\rangle$ and $\langle\langle+-|\mathcal{K}(\tau)|--\rangle$ (upper panel), $\langle\langle+-|\mathcal{K}(\tau)|+-\rangle$ (middle panel), and $\langle\langle+-|\mathcal{K}(\tau)|-+\rangle$ (lower panel), for a TLS coupled to a linear chain of 11 helium atoms. Note that $\langle\langle+-|\mathcal{K}(\tau)|++\rangle\rangle=\langle\langle+-|\mathcal{K}(\tau)|--\rangle\rangle$ at the WC limit.

the calculation of the memory kernel will lead to more accurate results.

- The WC and LHA-LSC-GQME treatments lead to significantly different asymptotic equilibrium states, which implies that the system-bath coupling cannot be assumed to be weak.

- One expects the direct LHA-LSC and WC relaxation behaviors to be overdamped and underdamped, respectively, relative to the exact result (cf. Sec. IV A). The damping in the relaxation predicted via the LHA-LSC-GQME method is indeed intermediate between those two extremes, which is consistent with the view that LHA-LSC-GQME is more accurate.

The LHA-LSC-based real and imaginary parts of $\langle\langle+-|\mathcal{K}(\tau)|++\rangle\rangle,\langle\langle+-|\mathcal{K}(\tau)|--\rangle\rangle,\langle\langle+-|\mathcal{K}(\tau)|+-\rangle\rangle$, and $\langle\langle+-|\mathcal{K}(\tau)|-+\rangle$ are shown in Figs. 8 and 9, respectively. Also shown in those figures are the corresponding predictions of the WC treatment, which are based on the bath-free $\mathrm{CF}$ in Fig. 6. The lifetimes of the GQME and WC memory kernels is seen to be comparable to that of the free bath $\mathrm{CF}$ in

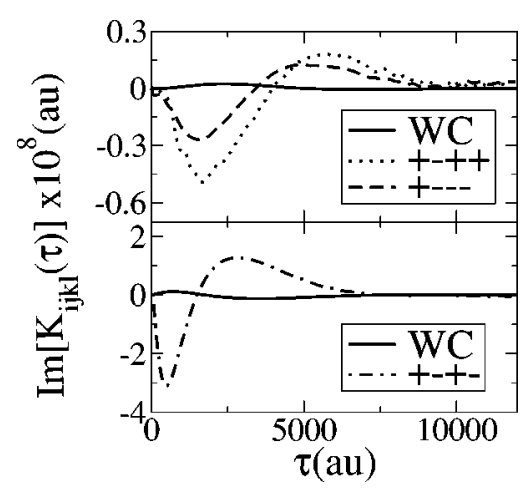

FIG. 9. The LHA-LSC-based and WC-based imaginary parts of $\langle\langle+-|\mathcal{K}(\tau)|$ $++\rangle\rangle$ and $\langle\langle+-|\mathcal{K}(\tau)|--\rangle\rangle$ (upper panel), and $\langle\langle+-|\mathcal{K}(\tau)|+-\rangle$ (lower panel), for a TLS coupled to a linear chain of 11 helium atoms. Note that $\operatorname{Im}\langle\langle+-|\mathcal{K}(\tau)|-+\rangle\rangle=0$ in this case (see lower panel of Fig. 8 for its real part). Note that $\langle\langle+-|\mathcal{K}(\tau)|++\rangle\rangle=\langle\langle+-|\mathcal{K}(\tau)|--\rangle\rangle$ at the WC limit. 
Fig. 6, and an order of magnitude smaller in comparison to the time scale of the TLS relaxation in Fig. 7. The GQME memory kernel is visibly different from its WC counterpart, which is consistent with the view that the WC limit is not valid in this case. Another signature to a non-WC behavior is provided by the difference between $\langle\langle+-|\mathcal{K}(\tau)|++\rangle\rangle$ and $\langle\langle+-|\mathcal{K}(\tau)|--\rangle\rangle$ (those two matrix elements coincide in the WC limit). ${ }^{78}$ As in the spin-boson case, all elements, except for $\langle\langle+-|\mathcal{K}(\tau)|+-\rangle$, vanish at $\tau=0$. This is consistent with the explicit expressions in the WC limit, and appears to remain valid beyond it.

Finally, we note that a few (less than $2 \%$ ) of the trajectories in the LHA-LSC calculation of the SDBCFs were observed to become unstable and were therefore discarded. This unphysical instability can be traced back to the MM mapping, which allows for initial values of $\sigma_{z}^{\mathrm{MM}}$ which are larger (smaller) than $1(-1)$, such that the system-bath coupling term, $\hat{H}_{\mathrm{bs}}=\hat{\Lambda} \hat{\sigma}_{z}$, can make the potential unbounded. It should be noted that the fact that the SDBCFs are relatively short lived plays a key role here too, since the number of unstable trajectories grows with time (for that reason, the direct LHA-LSC result in Fig. 7 is only given at relatively short times). We have verified that the SDBCFs obtained by discarding the unstable trajectories are in agreement with a calculation based on an alternative (as well as more computationally demanding) mixed quantum-classical Liouville treatment, which avoids those instabilities. A more detailed discussion of this point will be provided in a separate forthcoming paper.

\section{CONCLUSIONS}

In this paper, we proposed a new methodology for simulating the nonequilibrium reduced quantum dynamics of a system coupled to a bath. The new methodology is based on using the LSC approximation for calculating the two-time SDBCFs, which are then fed as input into the calculation of the memory kernel, and followed by propagation of the system density matrix based on the GQME. The new methodology can be used for simulating the quantum dynamics of strongly coupled and highly anharmonic condensed phase systems, even when it takes place far from equilibrium. As such, it represents an important step forward in our ability to simulate quantum dynamics beyond the domains of validity of LRT and WC treatments (cf. Fig. 1). The success of the new methodology relies on the ability of the LSC approximation to generate a reasonably accurate description of the dynamics over the life time of the memory kernel. Since the latter is typically much shorter than the system relaxation time, one expects the new methodology will generally be significantly more accurate in comparison to a direct application of the LSC approximation. The new methodology should also exhibit superior accuracy in comparison to standard QMEs of the Bloch-Redfield type, which are subject to the restrictive assumption of weak system-bath coupling and Markovity.

The LSC-GQME methodology presented herein is similar in spirit to the semiclassical methodology of Makri et al. for calculating the path integral IF that results from coupling to anharmonic environments. ${ }^{69,168,169}$ Similarly to the memory kernel, the IF provides a formally exact and compact parameterization of the influence of the bath on the system dynamics. The semiclassical approximation employed by Makri et al. is different from the LSC one, and is based on treating the forward-backward dynamics in terms of a single semiclassical propagator of the Herman-Kluk type. ${ }^{120}$ Self cancellations of the forward and backward actions then lead to a smoother integrand, which can be integrated over via MC techniques. This forward-backward semiclassical methodology is particularly suitable for calculating the IF, which is given by the trace over a product of real and imaginary time evolution operators. The application of the forward-backward semiclassical approach to the calculation of CFs of the form $\operatorname{Tr}\left(\hat{A} e^{i \hat{H} t / \hbar} \hat{B} e^{-i \hat{H} t / \hbar}\right)$ is also particularly straightforward when the operator $\hat{B}$ has an exponential form. Unfortunately, the SDBCFs may involve nonexponential operators. Although the latter may be represented in terms of exponential operators, the procedure is not unique, and different representations can lead to different results. ${ }^{127,151,170}$ In fact, it has been argued by Miller and co-workers that one of the most practical representations, which has been based on the identity $\hat{B}$ $=-\left.i(\partial / \partial \lambda) e^{i \lambda \hat{B}}\right|_{\lambda=0}$, Ref. 127, is closely related to the LSC approximation. ${ }^{170}$

A comparison of the computer memory requirements of the IF and GQME approaches seems to suggest that the latter provides a more favorable "packaging" of the information regarding the influence of the bath over the system's dynamics. More specifically, if $N$ is the number of states (e.g., corresponding to the states included in the discrete variable representation), and $k_{\max }$ is the number of time slices that the bath correlation time is divided into, then the computer space required for storing the IF scales like $N^{2 k_{\max }}$ (within the iterative tensor propagator method of Makri and co-workers), ${ }^{57-60,62,64}$ while the space required for storing the memory kernel scales like $\sim N^{4} \times k_{\max }$. This favorable scaling is intimately related to the fact that the memory kernel can be expressed in terms of $\sim N^{4}$ two-time CFs, whereas the IF is a function of the $2 k_{\max }$ variables that correspond to the discrete representation of the system's forward-backward path. Thus, it is easier to store the memory kernel in memory throughout the simulation in the case of a system with relatively large $N$ and $k_{\max }$. It should also be noted that the structure of the IF approach forces a description of the system dynamics in terms of path integrals, which may not be the most cost-effective framework for simulating the dynamics of what is usually a relatively small quantum system. At the same time, the description of the system dynamics via the GQME is formulated in terms of operators, and is therefore free of such constraints.

The LSC-GQME methodology presented herein would allow for the simulation of nonequilibrium quantum dynamics of a system with an arbitrary coupling to an anharmonic environment, and beyond the domains of LRT and WC treatments (see Fig. 1). Many exciting applications fall into this category, including electronic and vibrational relaxation, chromophore spectroscopy, coherent control, and chemical 
reactivity, in liquid solution and other anharmonic media. Those and other applications are the subject of ongoing work in our group, and will be reported in future publications.

\section{ACKNOWLEDGMENT}

This project was supported by the National Science Foundation FOCUS Center, through Grant No. PHY0114336.

${ }^{1}$ B. J. Berne, G. Ciccotti, and D. F. Coker, Classical and Quantum Dynamics in Condensed Phase Simulations (World Scientific, London, 1998).

${ }^{2}$ N. Makri, Annu. Rev. Phys. Chem. 50, 167 (1999).

${ }^{3}$ P. Jungwirth and R. B. Gerber, Chem. Rev. 99, 1583 (1999).

${ }^{4}$ R. Kosloff, J. Phys. Chem. 92, 2087 (1988).

${ }^{5}$ C. Leforestier, R. H. Bisseling, C. Cerjan et al., J. Comput. Phys. 94, 59 (1991).

${ }^{6} \mathrm{R}$. Kosloff, in Numerical Grid Methods and Their Application to Schrödinger's Equation, edited by C. Cerjan (Kluwer Academic, The Netherlands, 1993), p. 175

${ }^{7}$ R. Kosloff, Annu. Rev. Phys. Chem. 45, 145 (1994).

${ }^{8} \mathrm{R}$. Kosloff, in Dynamics of Molecules and Chemical Reactions, edited by D. T. Haar (Dekker, New York, 1996), p. 185.

${ }^{9}$ B. J. Berne and D. Thirumalai, Annu. Rev. Phys. Chem. 37, 401 (1986).

${ }^{10}$ D. M. Ceperley, Rev. Mod. Phys. 67, 279 (1995).

${ }^{11}$ R. Kubo, M. Toda, and N. Hashitsume, Statistical Physics II: Nonequilibrium Statistical Mechanics (Springer, New York, 1985).

${ }^{12}$ S. Mukamel, Principles of Nonlinear Optical Spectroscopy (Oxford, New York, 1995).

${ }^{13}$ T. Yamamoto, J. Chem. Phys. 33, 281 (1960).

${ }^{14}$ E. Geva, Q. Shi, and G. A. Voth, J. Chem. Phys. 115, 9209 (2001).

${ }^{15}$ K. Blum, Density Matrix Theory and Applications (Plenum, New York, 1996).

${ }^{16}$ F. Haake, Springer Tracts Mod. Phys. 66, 98 (1973).

${ }^{17}$ R. Alicki and K. Lendi, Quantum Dynamical Semigroups and Applications (Springer, Berlin, 1987).

${ }^{18}$ R. K. Wangsness and F. Bloch, Phys. Rev. 89, 728 (1953).

${ }^{19}$ A. G. Redfield, IBM J. Res. Dev. 1, 19 (1957).

${ }^{20}$ B. Yoon, J. M. Deutch, and J. H. Freed, J. Chem. Phys. 62, 4687 (1975).

${ }^{21}$ I. Oppenheim, K. E. Shuler, and G. H. Weiss, Stochastic Processes in Chemical Physics: The Master Equation (MIT Press, Cambridge, MA, 1977).

${ }^{22}$ S. Mukamel, I. Oppenheim, and J. Ross, Phys. Rev. A 17, 1988 (1978).

${ }^{23}$ V. Romero-Rochin and I. Oppenheim, Physica A 155, 52 (1989).

${ }^{24}$ V. Romero-Rochin, A. Orsky, and I. Oppenheim, Physica A 156, 244 (1989).

${ }^{25}$ N. G. van Kampen, Stochastic Processes in Physics and Chemistry (North-Holland, Amsterdam, 1981).

${ }^{26}$ B. B. Laird, J. Budimir, and J. L. Skinner, J. Chem. Phys. 94, 4391 (1991).

${ }^{27}$ W. T. Pollard and R. A. Friesner, J. Chem. Phys. 100, 5054 (1994).

${ }^{28}$ W. T. Pollard, A. K. Felts, and R. A. Friesner, Adv. Chem. Phys. XCIII, 77 (1996).

${ }^{29}$ E. Geva, R. Kosloff, and J. L. Skinner, J. Chem. Phys. 102, 8541 (1995).

${ }^{30}$ E. Geva and R. Kosloff, J. Chem. Phys. 104, 7681 (1996).

${ }^{31}$ D. Kohen, C. C. Marston, and D. J. Tannor, J. Chem. Phys. 107, 5236 (1997).

${ }^{32}$ J. Cao, J. Chem. Phys. 107, 3204 (1997)

${ }^{33}$ Y. J. Yan, Phys. Rev. A 58, 2721 (1998).

${ }^{34}$ M. Berman, R. Kosloff, and H. Tal-Ezer, J. Phys. A 25, 1283 (1992).

${ }^{35}$ G. Ashkenazi, U. Banin, A. Bartana, R. Kosloff, and S. Ruhman, Adv. Chem. Phys. 100, 229 (1997).

${ }^{36}$ G. Ashkenazi, R. Kosloff, and M. A. Ratner, J. Am. Chem. Soc. 121, 3386 (1999).

${ }^{37}$ R. Kosloff, M. A. Ratner, and W. B. Davis, J. Chem. Phys. 106, 7036 (1997).

${ }^{38}$ A. Suárez and R. Silbey, J. Chem. Phys. 94, 4809 (1991).

${ }^{39}$ D. Li and G. A. Voth, J. Phys. Chem. 95, 10425 (1991).

${ }^{40}$ B. B. Laird and J. L. Skinner, J. Chem. Phys. 94, 4405 (1991).

${ }^{41}$ D. R. Reichman and R. J. Silbey, J. Chem. Phys. 104, 1506 (1996).

${ }^{42}$ D. R. Reichman and F. L. H. B. P. Neu, Phys. Rev. E 55, 2328 (1997).

${ }^{43}$ S. Jang, J. Cao, and R. J. Silbey, J. Chem. Phys. 116, 2705 (2002).
${ }^{44}$ R. P. Feynman and A. R. Hibbs, Quantum Mechanics and Path Integrals (McGraw-Hill, New York, 1965).

${ }^{45}$ L. S. Schulman, Techniques and Applications of Path Integration (Wiley, New York, 1981)

${ }^{46} \mathrm{H}$. Kleinert, Path Integrals in Quantum Mechanics, Statistics and Polymer Physics (World Scientific, New Jersey, 1995).

${ }^{47}$ R. P. Feynman and F. L. Vernon, Jr., Ann. Phys. 24, 118 (1963).

${ }^{48}$ P. G. Wolynes, Phys. Rev. Lett. 47, 968 (1981).

${ }^{49}$ A. O. Caldeira and A. J. Leggett, Ann. Phys. 149, 374 (1983).

${ }^{50}$ R. D. Coalson, J. Chem. Phys. 86, 995 (1987).

${ }^{51}$ C. Mak and D. Chandler, Phys. Rev. A 44, 2352 (1991).

${ }^{52}$ M. Topaler and N. Makri, J. Chem. Phys. 101, 7500 (1994).

${ }^{53}$ M. Topaler and N. Makri, J. Chem. Phys. 97, 9001 (1992).

${ }^{54}$ M. Topaler and N. Makri, Chem. Phys. Lett. 210, 285 (1993).

${ }^{55}$ M. Topaler and N. Makri, Chem. Phys. Lett. 210, 448 (1993).

${ }^{56}$ M. Topaler and N. Makri, J. Phys. Chem. 100, 4430 (1996).

${ }^{57}$ D. E. Makarov and N. Makri, Chem. Phys. Lett. 221, 482 (1994).

${ }^{58}$ N. Makri and D. Makarov, J. Chem. Phys. 102, 4600 (1995).

${ }^{59}$ N. Makri and D. Makarov, J. Chem. Phys. 102, 4611 (1995).

${ }^{60}$ N. Makri, J. Math. Phys. 36, 2430 (1995).

${ }^{61}$ N. Makri, E. Sim, D. E. Makarov, and M. Topaler, Proc. Natl. Acad. Sci. U.S.A. 93, 3926 (1996).

${ }^{62}$ E. Sim and N. Makri, Comput. Phys. Commun. 99, 335 (1997).

${ }^{63}$ E. Sim and N. Makri, J. Phys. Chem. B 101, 5446 (1997).

${ }^{64}$ N. Makri, J. Phys. Chem. A 102, 4414 (1998).

${ }^{65}$ J. S. Shao and N. Makri, J. Chem. Phys. 116, 507 (2002).

${ }^{66}$ A. A. Golosov, R. A. Friesner, and P. Pechukas, J. Chem. Phys. 110, 138 (1999).

${ }^{67}$ A. A. Golosov, R. A. Friesner, and P. Pechukas, J. Chem. Phys. 112, 2095 (2000)

${ }^{68}$ N. Makri and K. Thompson, Chem. Phys. Lett. 291, 101 (1998)

${ }^{69}$ K. Thompson and N. Makri, J. Chem. Phys. 110, 1343 (1999).

${ }^{70}$ K. Thompson and N. Makri, Phys. Rev. E 59, R4729 (1999).

${ }^{71}$ S. Nakajima, Prog. Theor. Phys. 20, 948 (1958).

${ }^{72}$ R. Zwanzig, Lect. Theor. Phys. 3, 106 (1960).

${ }^{73}$ R. Zwanzig, J. Chem. Phys. 33, 1338 (1960).

${ }^{74}$ R. Zwanzig, Physica 30, 1109 (1984).

${ }^{75}$ I. Prigogine and P. Resibois, Physica 27, 629 (1961).

${ }^{76} \mathrm{H}$. Grabert, Projection Operator Techniques in Nonequilibrium Statistical Mechanics (Springer, Berlin, 1982).

${ }^{77}$ V. May and O. Kühn, Charge and Energy Transfer Dynamics in Molecular Systems (Willey, Berlin, 2000).

${ }^{78}$ Q. Shi and E. Geva, J. Chem. Phys. 119, 12045 (2003).

${ }^{79}$ G. D. Billing, Chem. Phys. Lett. 30, 391 (1975).

${ }^{80}$ G. D. Billing, J. Chem. Phys. 99, 5849 (1993).

${ }^{81}$ J. C. Tully and R. K. Preston, J. Chem. Phys. 55, 562 (1971).

${ }^{82}$ J. C. Tully, J. Chem. Phys. 93, 1061 (1990).

${ }^{83}$ P. J. Kuntz, J. Chem. Phys. 95, 141 (1991).

${ }^{84}$ A. I. Krylov, R. B. Gerber, M. A. Gaveau, J. M. Mestdagh, and B. Schilling, J. Chem. Phys. 104, 3651 (1996).

${ }^{85}$ K. Yamashita and W. H. Miller, J. Chem. Phys. 82, 5475 (1985).

${ }^{86}$ E. Rabani, G. Krilov, and B. J. Berne, J. Chem. Phys. 112, 2605 (2000).

${ }^{87}$ E. Sim, G. Krilov, and B. Berne, J. Phys. Chem. A 105, 2824 (2001).

${ }^{88}$ J. Poulsen and P. J. Rossky, J. Chem. Phys. 115, 8014 (2001).

${ }^{89}$ E. Gallicchio and B. J. Berne, J. Chem. Phys. 105, 7064 (1996).

${ }^{90}$ E. Gallicchio, S. A. Egorov, and B. J. Berne, J. Chem. Phys. 109, 7745 (1998).

${ }^{91}$ S. A. Egorov, E. Gallicchio, and B. J. Berne, J. Chem. Phys. 107, 9312 (1997).

${ }^{92}$ G. Krilov and B. J. Berne, J. Chem. Phys. 111, 9147 (1999).

${ }^{93}$ E. Rabani, D. R. Reichman, G. Krylov, and B. J. Berne, Proc. Natl. Acad. Sci. U.S.A. 99, 1129 (2002).

${ }^{94}$ A. A. Golosov, D. R. Reichman, and E. Rabani, J. Chem. Phys. 118, 457 (2003)

${ }^{95}$ G. A. Voth, Adv. Chem. Phys. 93, 135 (1996).

${ }^{96}$ Q. Shi and E. Geva, J. Chem. Phys. 116, 3223 (2002).

${ }^{97}$ J. Poulsen, S. R. Keiding, and P. J. Rossky, Chem. Phys. Lett. 336, 488 (2001).

${ }^{98}$ S. Jang, Y. Pak, and G. A. Voth, J. Phys. Chem. A 103, 10289 (1999).

${ }^{99}$ J. Cao and G. A. Voth, J. Chem. Phys. 100, 5106 (1994).

${ }^{100}$ J. Cao and G. A. Voth, J. Chem. Phys. 101, 6157 (1994).

${ }^{101}$ J. Cao and G. A. Voth, J. Chem. Phys. 101, 6168 (1994).

${ }^{102}$ S. Jang and G. A. Voth, J. Chem. Phys. 111, 2357 (1999).

${ }^{103}$ S. Jang and G. A. Voth, J. Chem. Phys. 111, 2371 (1999). 
${ }^{104}$ D. R. Reichman, P.-N. Roy, S. Jang, and G. A. Voth, J. Chem. Phys. 113, 919 (2000).

${ }^{105}$ A. Calhoun, M. Pavese, and G. A. Voth, Chem. Phys. Lett. 262, 415 (1996).

${ }^{106}$ U. W. Schmitt and G. A. Voth, J. Chem. Phys. 111, 9361 (1999).

${ }^{107}$ M. Pavese and G. A. Voth, Chem. Phys. Lett. 249, 231 (1996).

${ }^{108}$ K. Kinugawa, P. B. Moore, and M. L. Klein, J. Chem. Phys. 106, 1154 (1997).

${ }^{109}$ K. Kinugawa, P. B. Moore, and M. L. Klein, J. Chem. Phys. 109, 610 (1998).

${ }^{110}$ K. Kinugawa, Chem. Phys. Lett. 292, 454 (1998).

${ }^{111}$ M. Pavese, D. R. Bernard, and G. A. Voth, Chem. Phys. Lett. 300, 93 (1999).

${ }^{112}$ D. R. Reichman and E. Rabani, Phys. Rev. Lett. 87, 265702 (2001).

${ }^{113}$ E. Rabani and D. R. Reichman, J. Chem. Phys. 116, 6271 (2002).

${ }^{114}$ E. Rabani and D. R. Reichman, Phys. Rev. E 65, 036111 (2002).

${ }^{115}$ D. R. Reichman and E. Rabani, J. Chem. Phys. 116, 6279 (2002).

${ }^{116}$ H. Wang, X. Sun, and W. H. Miller, J. Chem. Phys. 108, 9726 (1998).

${ }^{117}$ E. Pollak and J. Liao, J. Chem. Phys. 108, 2733 (1998).

${ }^{118}$ W. H. Miller, Adv. Chem. Phys. 25, 69 (1974).

${ }^{119}$ W. H. Miller, J. Chem. Phys. 53, 3578 (1970).

${ }^{120}$ M. F. Herman and E. Kluk, Chem. Phys. 91, 27 (1984).

${ }^{121}$ E. J. Heller, J. Chem. Phys. 94, 2723 (1981).

${ }^{122}$ K. G. Kay, J. Chem. Phys. 100, 4377 (1994).

${ }^{123}$ M. Ovchinnikov and V. A. Apkarian, J. Chem. Phys. 105, 10312 (1996).

${ }^{124}$ M. Ovchinnikov and V. A. Apkarian, J. Chem. Phys. 108, 2277 (1998).

${ }^{125}$ X. Sun and W. H. Miller, J. Chem. Phys. 106, 916 (1997).

${ }^{126}$ W. H. Miller, Faraday Discuss. 110, 1 (1998).

${ }^{127}$ J. S. Shao and N. Makri, J. Phys. Chem. A 103, 7753 (1999).

${ }^{128}$ K. Thompson and N. Makri, Phys. Rev. E 59, R4729 (1999).

${ }^{129}$ O. Kühn and N. Makri, J. Phys. Chem. A 103, 9487 (1999).

${ }^{130}$ H. Wang, M. Thoss, and W. H. Miller, J. Chem. Phys. 112, 47 (2000).

${ }^{131}$ M. Ovchinnikov, V. A. Apkarian, and G. A. Voth, J. Chem. Phys. 184, 7130 (2001).

${ }^{132}$ W. H. Miller, J. Phys. Chem. A 105, 2942 (2001).

${ }^{133}$ N. Makri and W. H. Miller, J. Chem. Phys. 116, 9207 (2002).

${ }^{134}$ M. Hillery, R. F. O'Connell, M. O. Scully, and E. P. Wigner, Phys. Rep. 106, 121 (1984).

${ }^{135}$ K. Imre, E. Ozizmir, M. Rosenbaum, and P. F. Zweifel, J. Math. Phys. 8, 1097 (1967).

${ }^{136}$ I. Oppenheim and J. Ross, Phys. Rev. 107, 28 (1957).

${ }^{137}$ H. Mori, I. Oppenheim, and J. Ross, in Studies in Statistical Mechanics, edited by J. De Boer and G. E. Uhlenbeck (North-Holland, Amsterdam, 1962), Vol. 1.

${ }^{138}$ J. T. Hynes, J. M. Deutch, C. H. Wang, and I. Oppenheim, J. Chem. Phys. 48, 3085 (1968).
${ }^{139}$ E. J. Heller, J. Chem. Phys. 65, 1289 (1976).

${ }^{140}$ H. W. Lee and M. O. Scully, J. Chem. Phys. 73, 2238 (1980).

${ }^{141}$ R. E. Cline, Jr. and P. G. Wolynes, J. Chem. Phys. 88, 4334 (1988).

${ }^{142}$ V. Khidekecl, V. Chernyak, and S. Mukamel, in Femtochemistry: Ultrafast Chemical and Physical Processes in Molecular Systems, edited by M. Chergui (World Scientific, Singapore, 1996), p. 507.

${ }^{143}$ V. S. Filinov, Mol. Phys. 88, 1517 (1996).

${ }^{144}$ V. S. Filinov, Mol. Phys. 88, 1529 (1996).

${ }^{145}$ V. S. Filinov, S. Bonella, Y. E. Lazovik, A. V. Filinov, and I. Zacharov in Classical and Quantum Dynamics in Condensed Phase Simulations, edited by B. J. Berne, G. Ciccotti, and D. F. Coker (World Scientific, Singapore, 1997).

${ }^{146}$ J. Cao and G. A. Voth, J. Chem. Phys. 104, 273 (1996).

${ }^{147}$ R. Hernandez and G. A. Voth, Chem. Phys. 233, 243 (1998).

${ }^{148}$ X. Sun, H. Wang, and W. H. Miller, J. Chem. Phys. 109, 4190 (1998).

${ }^{149}$ X. Sun, H. Wang, and W. H. Miller, J. Chem. Phys. 109, 7064 (1998).

${ }^{150}$ H. Wang, X. Song, D. Chandler, and W. H. Miller, J. Chem. Phys. 110, 4828 (1999).

${ }^{151}$ X. Sun and W. H. Miller, J. Chem. Phys. 110, 6635 (1999).

${ }^{152}$ J. L. Liao and E. Pollak, J. Chem. Phys. 111, 7244 (1999).

${ }^{153}$ Q. Shi and E. Geva, J. Chem. Phys. 118, 8173 (2003).

${ }^{154}$ Q. Shi and E. Geva, J. Phys. Chem. A 107, 9059 (2003).

${ }^{155}$ Q. Shi and E. Geva, J. Phys. Chem. A 107, 9070 (2003).

${ }^{156}$ J. A. Poulsen, G. Nyman, and P. J. Rossky, J. Chem. Phys. 119, 12179 (2003).

${ }^{157}$ M. P. Miller and C. W. McCurdy, J. Chem. Phys. 69, 5163 (1978).

${ }^{158}$ C. W. McCurdy, H. D. Meyer, and W. H. Miller, J. Phys. Chem. 70, 3214 (1978).

${ }^{159}$ H. D. Meyer and W. H. Miller, J. Chem. Phys. 70, 3214 (1979).

${ }^{160}$ H. D. Meyer and W. H. Miller, J. Chem. Phys. 71, 2156 (1979).

${ }^{161}$ G. Stock and M. Thoss, Phys. Rev. Lett. 78, 578 (1997).

${ }^{162}$ M. Thoss and G. Stock, Phys. Rev. A 59, 64 (1999).

${ }^{163}$ A. J. Leggett, S. Chakravarty, A. T. Dorsey, M. P. A. Fisher, A. Garg, and W. Zwerger, Rev. Mod. Phys. 59, 1 (1987).

${ }^{164}$ U. Weiss, Quantum Dissipative Systems (World Scientific, London, 1993).

${ }^{165}$ W. H. Press, B. P. Flannery, S. A. Teukolsky, and W. T. Vetterling, $N u-$ merical Recipes (Cambridge University Press, Cambridge, 1986).

${ }^{166}$ E. Geva, E. Rosenman, and D. J. Tannor, J. Chem. Phys. 113, 1380 (2000).

${ }^{167}$ J. Poulsen and P. J. Rossky, J. Chem. Phys. 115, 8024 (2001).

${ }^{168}$ K. Thompson and N. Makri, Chem. Phys. Lett. 291, 101 (1998).

${ }^{169}$ N. Makri, J. Chem. Phys. 111, 6164 (1999).

${ }^{170}$ M. Thoss, H. Wang, and W. H. Miller, J. Chem. Phys. 114, 9220 (2001). 